\title{
To preparation of proposals for the target programs of development of housing and communal services
}

\author{
Rustam Khayrullin* \\ Moscow State University of Civil Engineering, Yaroslavskoe shosse, 26, Moscow, 129337, Russia
}

\begin{abstract}
The mathematical model of the formation and implementation of strategies for the development of the park of control and measuring equipment used in construction and housing and communal services is considered. As control functions, purchases, repairs and write-offs are selected. The model is based on a linear system of ordinary differential equations with constant coefficients. The system describes the dynamics of changes in the number of control and measuring equipment samples with different levels of technical excellence and technical condition. The model mainly uses algorithms based on the properties of stationary solutions of dynamic systems. Three different strategies for the development of a measuring equipment park have been considered. For each strategy, analytical and semi-analytical solutions of the stationary model are obtained. A distinctive feature of the model is its visibility and ease of use. The results of calculations are given.
\end{abstract}

\section{Introduction}

The park of control and measuring equipment (CME), used in the field of construction and housing and communal services, includes tens and even hundreds of thousands of CME samples. Over time, CME samples may degrade, may break (become faulty), may become morally and physically obsolete. Therefore, there is the problem of managing such a park of CME. Indicator of modernity and the indicator of serviceability are generalized indicators of park efficiency. The problem of managing the indices of modernity and serviceability of the park of CME is an urgent practical task not only in the sphere of housing and public utilities [1-5], but in the construction industry [6], in the field of engineering, in the field of aircraft building and other activities [7-9]. For the solution of the problem of effective controlling the park of CME there used variety of methods, for example [10-11].

\section{Mathematical model of transitions of state of samples of control and measuring equipment}

In this paper, we assume that according to the levels of technical excellence and technical condition CME samples are subdivided into modern serviceable, modern faulty, outdated

\footnotetext{
*Corresponding author: zrkzrk@list.ru
} 
serviceable and outdated faults. Let us denote: $x_{1}$ - number of modern serviceable CME samples; $x_{2}$ - number of modern faulty CME samples; $x_{3}$ - number of obsolete serviceable CME samples; $x_{4}$ - number of obsolete faulty CME samples. Consequintly $N=x_{1}+x_{2}+x_{3}+x_{4}$ - total number of CME samples.

The total number of CME samples in the park is determined by directive standards, the needs of production and operation, as well as tactical and strategic tasks of management companies.

Suppose that the following statistical values of the probabilities of transitions between the states of the CME samples were determined as a result of statistical data processing for a sufficiently long period of time:

$p_{12}$ - the probability of a transition from modern serviceable to modern faulty state;

$p_{13}$ - the probability of a transition from modern serviceable to obsolete serviceable state;

$p_{24}$ - the probability of transition from a modern faulty state to an obsolete faulty state;

$p_{34}$ - the probability of a transition from the obsolete serviceable state to obsolete faulty state. controls:

We describe other transitions of states, which in the present paper we consider as $u_{21}$ - repair of modern faulty samples;

$u_{43}$ - repair of obsolete faulty samples;

$u_{4}$ - procurement of modern serviceable samples;

$u_{6}$ - discarding of obsolete faulty samples;

$k_{41}$ - the share of outdated faulty samples, instead of which the purchase of modern serviceable samples to be made;

$k_{43}$ - the share of obsolete faulty samples to be repaired;

$k_{21}$ - the share of modern faulty samples to be repaired.

We will asume that after repair and verification faulty samples become serviceable.

The graph of state transitions for $k_{21}=u_{21}, k_{43}=u_{43}, k_{41}=u_{6}=u_{4}$ is shown in Fig. 1a). The graph of state transitions for $u_{4} \neq u_{6}$ is shown in Fig.1b).
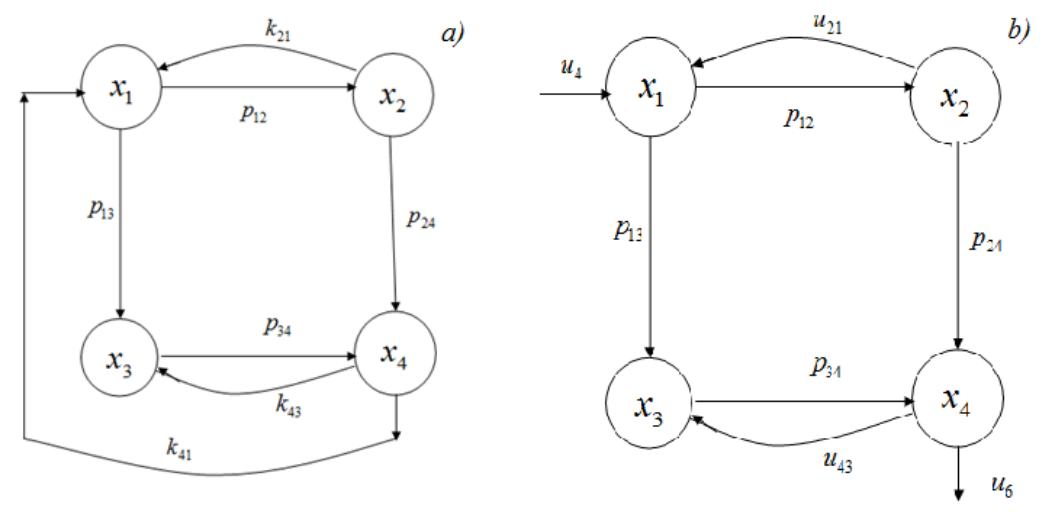

Fig. 1. Graph of state transitions of CME: a) for $u_{4}=u_{6}$; b) for $u_{4} \neq u_{6}$. 
Dynamic equations $[5,10]$ describing the process of development of the CME park are:

$$
\left\{\begin{array}{l}
\frac{d x_{1}}{d t}=-p_{12} x_{1}-p_{13} x_{1}+k_{21} x_{2}+k_{41} x_{4} \\
\frac{d x_{2}}{d t}=-p_{24} x_{2}+p_{12} x_{1}-k_{21} x_{2} \\
\frac{d x_{3}}{d t}=-p_{34} x_{3}+p_{13} x_{1}+k_{43} x_{4} \\
\frac{d x_{4}}{d t}=p_{24} x_{2}+p_{34} x_{3}-k_{41} x_{4}-k_{43} x_{4}
\end{array}\right.
$$

We will assume that the initial conditions are given: $x_{i}(0)=x_{i}^{z},(i=1,2,3,4)$.

If the coefficients of the variables $\left(x_{i}, i=1,2,3,4\right)$ in the right-hand sides of the equations are constant, then system (1) has a stationary solution, to which all particular solutions of the system aspire to $t \rightarrow \infty$.

It is required to find such values of the control parameters $k_{41}, k_{43}, k_{21}$ at which the indicators of modernity $P_{c}=\left(x_{1}+x_{2}\right) / N$ and serviceability $P_{u}=\left(x_{1}+x_{3}\right) / N$ in the long term (when $t \rightarrow \infty$ ) will take the required predetermined values.

A stationary solution can be found by equating the derivatives on the left side of the system (1) to zero. The stationary solution satisfies the following system of linear homogeneous algebraic equations for unknown variables $x_{1}, x_{2}, x_{3}, x_{4}$ :

$$
\left\{\begin{array}{l}
-p_{12} x_{1}-p_{13} x_{1}+k_{21} x_{2}+k_{41} x_{4}=0 \\
-p_{24} x_{2}+p_{12} x_{1}-k_{21} x_{2}=0 \\
-p_{34} x_{3}+p_{13} x_{1}+k_{43} x_{4}=0 \\
p_{24} x_{2}+p_{34} x_{3}-k_{41} x_{4}-k_{43} x_{4}=0
\end{array}\right.
$$

The rank of system (2) is equal to three. If as the free variable we chose $x_{2}$, then the general solution (2) can be represented as:

$$
\left\{\begin{array}{l}
x_{1}=\frac{p_{24}+k_{21}}{p_{12}} \cdot x_{2} \\
x_{3}=\frac{p_{13} k_{41}\left(p_{24}+k_{21}\right)+k_{43}\left(p_{12} p_{24}+p_{13} p_{24}+p_{13} k_{21}\right)}{p_{34} k_{41} p_{12}} \cdot x_{2} \\
x_{4}=\frac{p_{12} p_{24}+p_{13} p_{24}+p_{13} k_{21}}{k_{41} p_{12}} \cdot x_{2}
\end{array} .\right.
$$




\section{Development of strategies for the development of CME park}

We describe three different strategies for the development of CME park.

\subsection{Strategy 1}

We consider the case $k_{43}=0, k_{21} \neq 0, k_{41} \neq 0$, which corresponds to the strategy, when a certain proportion of modern faulty CME samples are subject to repair and verification, outdated faulty CME samples cannot be repaired, instead of them modern serviceable samples can be purchased. Then from (3) it follows:

$$
\begin{gathered}
x_{1}=\frac{p_{24}+k_{21}}{p_{12}} \cdot x_{2}, \quad x_{3}=\frac{p_{13} k_{41}\left(p_{24}+k_{21}\right)}{p_{34} k_{41} p_{12}} \cdot x_{2}, \\
x_{4}=\frac{p_{12} p_{24}+p_{13} p_{24}+p_{13} k_{21}}{k_{41} p_{12}} \cdot x_{2} .
\end{gathered}
$$

Restriction on total number of CME, as well as restrictions on the indices of modernity and serviceability, we write in the form of a system of equations:

$$
\left\{\begin{array}{l}
x_{1}+x_{2}+x_{3}+x_{4}=N \\
x_{1}+x_{2}=N \cdot P_{c} \\
x_{1}+x_{3}=N \cdot P_{u}
\end{array},\right.
$$

where $P_{c}$ - indicator of the modernity, $P_{u}$ - indicator of the serviceable of the CME park. If in the left part of (5) we substitute the expression (4), then the left parts of relations (5) will depend on the unknown parameters $k_{41}, k_{21}$ and variable $x_{2}$. Solving this system of nonlinear algebraic equations by the Newton method [11], we obtain the values $k_{41}$ and $k_{21}$, providing the required level of modernity and serviceability of the CME park.

Note that the order of system (5) can be reduced to the second order, if we express the variable $x_{2}$ from the first relation (5) with regard to (4) and substitute (6) into the second and third relation $(5)$ :

$$
x_{2}=N / Z_{1}
$$

where

$$
Z_{1}=\frac{p_{24}+k_{21}}{p_{12}}+\frac{p_{13} k_{41} p_{24}+p_{13} k_{41} k_{21}}{p_{34} k_{41} p_{12}}+\frac{p_{12} p_{24}+p_{13} p_{24}+p_{13} k_{21}}{k_{41} p_{12}}+1
$$

\subsection{Strategy 2}

Let us now consider a special case $k_{21}=0, k_{41} \neq 0, k_{43} \neq 0$, which corresponds to the strategy when modern faulty CME samples cannot be repaired, some of the obsolete faulty CME samples are subject to repair and verification, instead of some other part of the obsolete faulty samples, modern serviceable samples are purchased. Then from (3) it follows: 


$$
\begin{gathered}
x_{1}=\frac{p_{24}}{p_{12}} \cdot x_{2}, \quad x_{3}=\frac{p_{13} k_{41} p_{24}+k_{43} p_{12} p_{24}+k_{43} p_{13} p_{24}}{p_{34} k_{41} p_{12}} \cdot x_{2}, \\
x_{4}=\frac{p_{12} p_{24}+p_{13} p_{24}}{k_{41} p_{12}} \cdot x_{2} .
\end{gathered}
$$

Restrictions on the total number of CME, as well as the limitations on the indicators of modernity and serviceability are (5).

The left parts of (5) subject to condition (7) depend on the unknown parameters $k_{41}$, $k_{43}$ and variable $x_{2}$. Solving this system by the Newton method, we obtain $k_{41}, k_{43}$ providing the required level of modernity and serviceability of the CME park.

Note that the order of system (5) can be reduced to the second order, if we express the variable $x_{2}$ from the first equation (5) taking into account (7) and substitute (8) into the second and third relations (5):

$$
x_{2}=N / Z_{2},
$$

where

$$
Z_{2}=\frac{p_{24}}{p_{12}}+\frac{p_{13} k_{41} p_{24}+k_{43} p_{12} p_{24}+k_{43} p_{13} p_{24}}{p_{34} k_{41} p_{12}}+\frac{p_{12} p_{24}+p_{13} p_{24}}{k_{41} p_{12}}+1
$$

Resulting values of the control parameters must satisfy the constraints: $0 \leq k_{21} \leq 1$, $0 \leq k_{43} \leq 1, \quad 0 \leq k_{41}+k_{43} \leq 1, \quad\left(x_{i} \geq 0, i=1,2,3,4\right)$. If, as a result of solving a system of nonlinear equations, the values of the control parameters are obtained that do not satisfy the indicated constraints, then this indicates that the control resource is not sufficient for the simultaneous fulfillment of requirements for modernity and good condition. In this case, to obtain a result suitable for practical use, it is necessary to attract additional resources. For example, joint repair and verification of both modern faulty and obsolete faulty CME samples. One of these possible strategies is described below.

We also note that in some cases the simultaneous fulfillment of restrictions on modernity and serviceability indices in the form of two independent equalities turns out to be impossible. Then it is recommended to weaken one of the restrictions and take it into account in the form of inequality.

\subsection{Strategy 3}

Let us consider a special case $x_{2}=0, k_{21} \neq 0, k_{41} \neq 0, k_{43} \neq 0$, corresponding to the strategy, when all modern faulty samples are subject to repair and verification in the same planning interval in which they become faulty, and also part of the obsolete faulty CME samples is subject to repair and verification. In addition, instead of a portion of obsolete faulty samples, modern, serviceable samples are purchased.

The stationary solution satisfies the following system of linear homogeneous algebraic equations: 


$$
\left\{\begin{array}{l}
-p_{13} x_{1}+k_{41} x_{4}=0 \\
-p_{34} x_{3}+p_{13} x_{1}+k_{43} x_{4}=0 \\
p_{34} x_{3}-k_{41} x_{4}-k_{43} x_{4}=0
\end{array}\right.
$$

The rank of the system (9) is equal to two. If we choose as a free variable $x_{4}$, then the general solution of the system can be represented as:

$$
x_{1}=\frac{k_{41}}{p_{13}} \cdot x_{4}, x_{3}=\frac{k_{41}+k_{43}}{p_{34}} \cdot x_{4} .
$$

The restrictions on the total number of CME samples, as well as the restrictions on the indices of modernity and serviceability, still have the form (5).

The left parts of the equations depend on unknown values $k_{41}, k_{43}$. So we get from (10) and the first constraint (5):

$$
x_{4}=\frac{N}{\frac{k_{41}}{p_{13}}+\frac{k_{41}+k_{43}}{p_{34}}+1} .
$$

If we substitute expression (11) in the second and third equation (5), then we obtain a system of second-order linear algebraic equations for $k_{41}, k_{43}$. The explicit solution of this system is:

$$
k_{41}=\frac{P_{c} p_{13}}{1-P_{u}}, k_{43}=\frac{P_{u} p_{34}-P_{c}\left(p_{34}+p_{13}\right)}{1-P_{u}} .
$$

The obtained analytical dependencies (12) allow us to calculate the values of the control parameters $k_{41}$ and $k_{43}$, depending on the required values of the performance indicators $P_{c}$ and $P_{u}$. Fig. 2a,b shows the corresponding two-dimensional dependences.
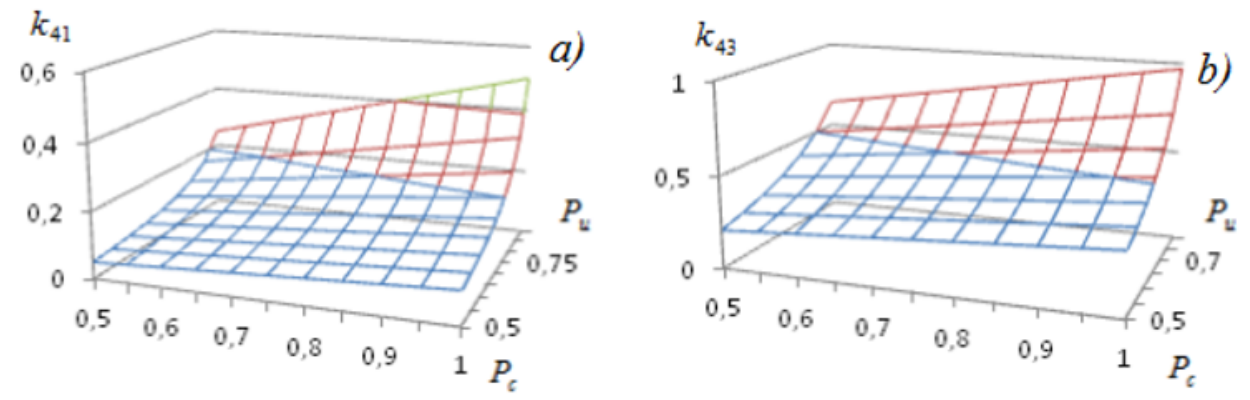

Fig. 2. Dependences of control parameters ( $k_{41}$ - shares of purchased CME samples and $k_{43}$ shares of repaired CME samples) on target values of indices of modernity and serviceability. 
Let us note that with the increasing of performance indices in the required values, the share of purchased and repaired CME samples increases too. Note that the dependencies obtained in this work have the same behavior as the dependencies [4,5]

\section{Discussions}

This paper presents the mathematical model for the task of targeted planning for the development of the sphere of housing and public utilities based on the application of stationary solution of continuous dynamic model. The [4] presents the results of solving a similar problem based on the use of a discrete dynamic model. These mathematical models (dynamic continuous model and dynamic discrete model) form the basis of the software for the development of proposals for targeted development programs in the construction and housing and utilities sectors. Depending on the objectives of the study and the specific problem being solved, one or another mathematical model can be used.

The mathematical model developed in this paper should be used in the long-term development planning of the CME park, which includes a large and very large number of CME samples. At the same time, model [4,5] is more adequate when planning the development of a medium-sized park with a relatively small CME nomenclature.

\section{Conclusions}

The main results of the work are as follows:

1. The mathematical model has been developed that describes the development of CME park based on the properties of stationary solutions of dynamic systems.

2. Algorithms for calculating controlled parameters providing the target indices values of development of the CME park were created.

3. The solution of the problem of program - target management of indices of efficiency of CME park in the form of synthesis was obtained. Explicit formulas are derived that allow to calculate the values of the control parameters necessary to achieve the target values of the indices of modernity and availability.

4. The properties of stationary solutions of the dynamic model of the CME park development are studied. The properties allow to understand the qualitative patterns of the dynamics of the CME park development and the limiting possibilities of CME park management. It is shown that the control parameters and performance indices satisfy the system of nonlinear algebraic equations. This system of equations can be effectively solved by the Newton's method [11].

\section{References}

1. Jas'kova N and Karasik D 2014 Vestnik MGSU 9

2. Khayrullin R 2017 On Preparing Offers for Targeted Development Programs in Housing and Communal Services. MATEC Web Conf 11700076

3. Marichev P, Kornev A and Khayrullin R 2018 The program - target planning and management by performance indicators of measuring equipment park Vestnik MGSU 13 pp 564-571.

4. Khayrullin R and Ivanov P 2018 Step-by-step control of target efficiency indices of the control and measuring equipment stock applied in construction and housing and communal services MATEC Web Conf $\mathbf{1 7 0} 01010$

5. Khayrullin R, Zubkov S and Lutskova T 2018 Systems Methods and Technologies 4 (38) pp 85-90 
6. Antonov A 2010 Transport business in Russia $\mathbf{1 4}$

7. Tuskaeva Z 2016 Formation of centers of technical equipment construction Vestnik $M G S U, 9$, pp 75-85

8. Lifanov I and Sherstjukov N G 1979 Metrology, tools and methods of quality control in construction (Moscow, Stroyizdat Pub) p 225

9. Akberdin R 1987 Economics of updating of Park of equipment in mechanical engineering (Moscow, Mashinostroenie Pub) p 47

10. Ventzel E 1972 Operations Research (Moscow, Science Pub) p 552

11. Fedorenko R 1978 Approximate solution of optimal control problems (Moscow, Science Pub) p 488 